\title{
ARTÍCULO
}

\section{Tecnología digital: ¿realidad aumentada o deformada?}

\section{Digital Technology: Distorted or Augmented Reality?}

\author{
Alfonso Ballesteros \\ Departamento de Ciencia Jurídica \\ Universidad Miguel Hernández de Elche
}

Fecha de recepción 26/12/2019 | De aceptación: 20/04/2020 | De publicación: 15/06/2020

\section{RESUMEN}

Este trabajo pretende ofrecer una aproximación humanista a la tecnología digital alrededor de las preguntas: “¿la tecnología digital aumenta o disminuye la estatura del hombre?”, “¿mejora la realidad o la deforma?". Se sostiene aquí que, fundamentalmente, deforma la realidad y la existencia humanas. Así, deforma progresivamente el conocimiento humano (deformación epistemológica), al individuo (deformación antropológica) y la relación con los otros (deformación socio-política). Quizá fijarnos en el modelo del jardinero, por su conocimiento sensorial, su paciencia y su capacidad de distinguir lo dado de lo construido pueda apuntar una salida a este problema.

\section{PALABRAS CLAVE}

tecnología digital, sentido común, libertad de expresión, libertad de multiplicación, economía de la atención, tecnologías de búsqueda, tecnologías de la reputación

\section{ABSTRACT}

Abstract: This paper aims to offer a humanist approach to digital tecnology by asking: "Does digital technology increase or disminish the stature of man?", “does it offer us a better reality or does it distort it?” Here I consider that digital tecnology distorts reality. Distorts human knowledge (epistemological distortion), the individual (antropological distortion) and the relationship with others (socio-political distortion). It might be a lead out to look at the gardener with his sense-based-knowledge, his pacience and his capacity to distinguish between what is given and what is built.

\section{KEY WORDS}

digital technology, common sense, freedom of speech, free reach, attention economy, seach technologies, reputation technologies 
SUMARIO: 1. Introducción. 2. ¿La tecnología digital aumenta o disminuye la estatura del hombre? 3. Deformación epistemológica. 3.1. La realidad deformada. 3.2. La pérdida del sentido común: tecnologías de búsqueda y de la reputación. 3.3. Información formal frente a conocimiento y sabiduría. 4. Deformación antropológica y política. 4.1. Economía de la atención: expropiación, adicción y transparencia. 4.2. ¿Libertad de expresión o multiplicación gratuita? 4.3. ¿Inteligibilidad o cajas negras? 5. A modo de conclusiones. 6. Bibliografía.

\section{Introducción}

Con este trabajo pretendo sostener que la digitalización actual se caracteriza por la deformación de la realidad. Hay dos tipos de tecnologías que contribuyen a esto. Por un lado, las "tecnologías de búsqueda" que configuran con sus algoritmos cómo percibimos el mundo. Presentan al usuario una realidad personalizada y son adictivas por diseño. Por otro lado, las "tecnologías de reputación” dan forma a cómo somos percibidos por otros. Son tecnologías propias de la llamada sociedad de la puntuación y crean las nuevas clases sociales.

La deformación de la realidad opera contra los sentidos, por lo que el sentido común no parece poder desarrollarse, pero tampoco un conocimiento racional. Por un lado, la tecnología digital es un medio emocional que impide el conocimiento reflexivo. Por otro, la capacidad de predicción algorítmica pretende hacer innecesaria la reflexión teórica y toda pregunta teleológica. El dominio de la probabilidad algorítmica de los dígitos, además, homogeneiza el conjunto de la realidad y hace al individuo cada vez más indistinguible de su entorno, pues su percepción del mundo y su reputación están presentadas por algoritmos, unificadas por información estructurada matemáticamente.

La tecnología digital no solo deforma la posibilidad de conocer, también deforma al individuo y su relación con los otros. Esta tecnología parece tener un carácter fuertemente parasitario, se beneficia de la misma vida del usuario que es convertida en producto. Con este fin esta tecnología es adictiva y pretende captar la atención absoluta del individuo y recabar toda la información sobre él. Luego la información es vendida a brókers de datos al tiempo que sirve de objeto de aprendizaje para la máquina, que mejora progresivamente con más información. Como la finalidad es explotar la vida humana por completo los algoritmos dan primacía y multiplican todo contenido que optimice el tiempo 
de exposición tecnológica de los usuarios. Así, se multiplica la información que sirve a este fin, aunque sea información falsa, pornográfica o que polarice a la sociedad. Por lo anterior, la tecnología digital impide la genuina política y permite una manipulación sin precedentes a escala global.

Detrás de todo este entramado de la realidad deformada operan algoritmos incomprensibles por el lego, una incomprensión que blinda el dominio de las tecnológicas y permite una mayor admiración hacia ellas pues estas parecen tener una mágica capacidad de predicción y un enorme poder de agradar al usuario al presentarle una realidad deformada a su gusto.

\section{2. ¿La tecnología digital aumenta o disminuye la estatura del hombre?}

El punto de partida de esta reflexión es que la neutralidad de la tecnología en sentido estricto es imposible. Ningún instrumento, ninguna tecnología es neutra. McLuhan, en su conocida frase afirma que "el medio es el mensaje". Es decir, el medio tiene una estructura ${ }^{1}$, una naturaleza. También su discípulo, Neil Postman, ha mostrado que toda tecnología está dotada de una estructura, no es una tabula rasa. Por ejemplo, el ordenador favorece el aprendizaje privado y la resolución individual de problemas frente al aprendizaje oral que es cooperativo ${ }^{2}$.

Otro ejemplo es la aparición de la escritura que influye de forma significativa en el ser humano que hace uso de ella con independencia del contenido de lo que se escriba. La estructura de la tecnología y el uso concreto que se hace de ella son cosas distintas. En la vieja fábula del Fedro, el rey Thamus se muestra escéptico ante la escritura porque está en la naturaleza de la escritura que la memoria se vuelva de mucha menos relevancia y, por ello, merme la capacidad memorística del ser humano con independencia de lo que escriba ${ }^{3}$. En esta línea y, en consecuencia, el diseño de cosas es una actividad moral ${ }^{4}$ y más moral cuanto más estructurada o más persuasiva sea la tecnología. Desde un punto de vista moral la estructura señala la responsabilidad del diseñador, la ambivalencia del uso, la del usuario. A menor ambivalencia, mayor responsabilidad y poder del diseñador.

\footnotetext{
${ }^{1}$ McLUHAN, M.; Comprender los medios de comunicación. Las extensiones del ser humano (trad. P. Ducher), Barcelona, Paidós, 1996, pp. 29ss.

2 POSTMAN, N.; Tecnópolis. La rendición de la cultura a la tecnología (trad. A. Almazán y S. Miras), El salmón, 2018 , pp. $23-37$.

${ }^{3}$ Ibid., pp. 19-40.

${ }^{4}$ VERBEEK, P.-P.; Understanding and Designing the Morality of Things, University of Chicago Press, 2011, pp. 22-23.
} 
La tecnología digital actual busca persuadir, es adictiva por diseño. Esto da respuesta a una lucrativa economía que explota nuestra atención. Es una tecnología cada vez más estructurada, en el sentido de que condiciona cada vez más al usuario que la emplea. No pretende ser una simple herramienta sino promover la dirección de la acción, por ejemplo, el envío de contenidos de forma masiva gracias al botón de compartir con un clic. La ambivalencia se reduce casi por completo o, peor, es ilusoria, pues el usuario hace lo que el diseñador ha previsto incluso cuando hace lo que cree desear por sí mismo. Este dominio de la psique mediante la técnica ha sido denominado acertadamente "psicopolítica"5. La tecnología es adictiva por diseño en un nivel pre-reflexivo pues no deja tiempo a la razón a manifestarse.

Las implicaciones ético-jurídicas de lo anterior son múltiples. Si la tecnología es persuasiva y condiciona profundamente la acción, el diseñador se sitúa como agente fundamental. Nuestro derecho ha caído en el error de asumir la neutralidad, por ejemplo, del buscador de Google, respecto de los contenidos que ofrece ${ }^{6}$. Por fortuna, se empieza a abandonar esta mentalidad que priva de responsabilidad a las empresas tecnológicas. El Reglamento general de protección de datos personales, de reciente entrada en vigor en la UE, da esperanza en la medida en que acude a una de las causas del problema de esta deformación de la realidad: la anomia e irresponsabilidad de los ingenieros y diseñadores. El Reglamento les exige garantizar la protección de datos desde el diseño y por defecto. Así, el Reglamento acude a una de las raíces del problema. Sin embargo, hay otros bienes humanos dañados por la tecnología digital, junto con la intimidad y los datos personales, por ello es necesario un enfoque más amplio.

Si la tecnología no es neutra cabe hacer la pregunta que hacía la humanista Hannah Arendt ante los avances en la conquista del espacio el siglo pasado, adaptándola al problema actual: "¿La tecnología digital aumenta o disminuye la estatura del hombre?" No se refiere a la estatura del consumidor, productor o diseñador, sino del hombre como tal. Tristan Harris considera que la respuesta

\footnotetext{
${ }^{5}$ HAN, B.-C.; Psicopolitica. Neoliberalismo y nuevas técnicas de poder (trad. A. Bergés), Barcelona, Herder, 2014.

${ }^{6}$ La Ley 34/2002 de 11 de julio de Servicios de la Sociedad de la Información y de Comercio Electrónico (LSSI), artlos. 14-17 es sumamente protectora. La preocupación de la LSSI (y la Directiva 2000/31/CE que traspone) era la propiedad intelectual, un enfoque completamente obsoleto ante el alcance de los problemas actuales.
} 
es clara: la disminuye y degrada ${ }^{7}$. Ciertamente, la tecnología digital está determinada por la economía de la explotación de la atención cuyo interés principal es la captación absoluta de la atención del individuo, apartándolo de la realidad analógica ${ }^{8}$. Este es el primer punto que se trata aquí, que afecta al conocimiento humano, lo que puede considerarse como una deformación epistemológica.

\section{Deformación epistemológica}

\subsection{La realidad deformada}

“Convertirán los ordenadores el egocentrismo en una virtud?”9 se preguntaba Postman hace casi tres décadas. Es claro que la tecnología digital nos aísla, nos hace estar "solos juntos" en expresión de Turkle ${ }^{10}$. Si nos hace estar solos estando con otros es porque nos aparta del aquí (de los otros que están aquí) y lo sustituye por el ahora (quizá, por otros que están lejos). Como ya vio Virilio, supone "negar el "aquí" en beneficio del "ahora""11.

El acercamiento a la tecnología nos aparta de los demás, pero también de toda la realidad, una realidad que se difumina. La tecnología digital busca eliminar el principio de realidad al tiempo que multiplica el presente ${ }^{12}$. El ahora se agranda de forma extraordinaria dando una impresión de omnipotencia al usuario en su disposición sobre el presente, ominpotencia reforzada por el dominio de lo táctil ${ }^{13}$. En esta realidad deformada se confunde progresivamente lo real con lo virtual y toda distinción ontológica entre individuos, animales y cosas se diluye $\mathrm{e}^{14}$.

El homo digitalis es, de forma creciente, un individuo que se vuelve hacia su cómodo mundo particular, como alguien que está dormido, pero que carece de mundo común, que es el de los que están

\footnotetext{
${ }^{7}$ CENTER FOR HUMANE TECNOLOGY; "Down the Rabbit Hole by Dessign”, Your Undivided Attention, Entrevista a Guillaume Chaslot, 2019, p. 17.

${ }^{8}$ WU, T.; The Attention Merchants' Dissects the Battle for Clicks and Eyeballs, Random House, 2016.

${ }^{9}$ POSTMAN, N.; Tecnópolis, cit., p. 37.

10 TURKLE, S.; Alone Together. Why We Expect More from Technology and Less from Each Other, New York, Basic Books, 2011. VIRILIO, P.; El cibermundo, la política de lo peor (Entrevista con Philippe Petit, trad. M. Poole), Madrid, Cátedra, 1997, p. 86. En contra: FLORIDI, L. (ed.); "Background Document: Rethinking Spaces in the Digital Transition", en The Onlife Manifesto. Being Human in a Hyperconnected Era, Springer Open, 2015, p. 46.

${ }^{11}$ VIRILIO, P.; El cibermundo, la politica de lo peor, cit., p. 46.

12 SADIN, E.; La humanidad aumentada (trad. J. Blanco y C. Paccazochi), Buenos Aires, Caja negra, 2017, pp. 84-85.

${ }^{13}$ SADIN, E.; La silicolonización del mundo. La irresistible expansión del liberalismo digital (trad. M. Martínez,), Buenos Aires, Caja negra, 2018, p. 233.

${ }^{14}$ FLORIDI, L. (ed.); The Onlife Manifesto, cit., pp. 43-46.
} 
despiertos ${ }^{15}$. Efectivamente, la digitalización supone la configuración de una nueva realidad altamente personalizada gracias a la matematización de la vida ${ }^{16}$.

La denominada "realidad aumentada" intenta suplir nuestras supuestas deficiencias sensoriales y cognitivas (ya no pretende aumentar nuestros sentidos ${ }^{17}$, sino sustituirlos), como las gafas de Google que nos presentan una realidad distorsionada ${ }^{18}$. Estas gafas destruyen la vista al transformar el mirar en información, un mirar matematizado que es como el mirar de un cíborg. La visión del mundo de la máquina no es una visión humana que permite conocer, es una visión deformada.

Se pueden presentar tres ejemplos de realidad deformada. El primero se refiere a la belleza de la realidad, el segundo a la confianza en la realidad y, el tercero, a la personalización de la realidad. El primero es el síndrome de París. Es el caso de los turistas japoneses que visitan París y les produce una enorme decepción porque la ciudad real es inferior a la de la imagen digital ${ }^{19}$. El segundo es la tragedia de la mujer que confió más en su GPS que en el cartel que indicaba que no cabía continuar por la carretera $^{20}$. El tercero tuvo lugar cuando British Petroleum dominaba la atención en las noticias de la televisión estadounidense en el verano de 2010. En aquel momento los resultados de las búsquedas "BP" en Google ofrecían a algunos usuarios enlaces para denunciar con contundencia las consecuencias para el medio ambiente de su actividad, mientras que para otros los enlaces conducían a oportunidades de inversión en la compañía ${ }^{21}$.

\subsection{La pérdida del sentido común: tecnologías de búsqueda y de la reputación}

Los tres ejemplos ponen de manifiesto esta sustitución de los sentidos y de la realidad por algo distinto, una realidad deformada y personalizada que destruye el mundo común. Frente a esta tendencia

\footnotetext{
15 DEWANDRE, N.; “The Human Condition and The Black Box Society”, https://www.boundary2.org/2015/12/dewandre-on-pascal/ [consultado: 13/12/2019].

${ }^{16}$ SADIN, E.; La humanidad aumentada, cit., p. 87.

${ }^{17}$ McLUHAN, M.; Comprender los medios de comunicación, cit., pp. 29ss.

${ }^{18}$ PASQUALE, F.; "Platform Neutrality: Enhancing Freedom of Expression in Spheres of Private Power", Theoretical Inquiries in Law, vol. 17, 2016, p. 512.

${ }^{19}$ Véase HAN, B.-C.; En el enjambre (trad. R. Gabás), Barcelona, Herder, 2014, p. 35.

${ }^{20}$ MORATALLA, N.; Inteligencia artificial. ¿Conciencia artificial? Madrid, Digital Reasons, 2017, p. 38.

${ }^{21}$ PARISER, E.; El filtro burbuja. Cómo la red decide lo que leemos y lo que pensamos (trad. M. Vaquero), Taurus, 2017, introducción. PASQUALE, F.; The Black Box Society. The Secret Algorithms That Control Money and Information, Cambridge-MAS, Harvard University Press, 2015, p. 79.
} 
recordemos a Hannah Arendt y su reivindicación del sentido común. Nuestro conocimiento del mundo adquiere certeza, señala, porque es un conocimiento compartido:

"nuestra certeza de que lo que percibimos tiene una existencia independiente del acto de la percepción, depende enteramente del hecho de que el objeto también aparece como tal ante otros, que así lo reconocen. Sin este reconocimiento tácito por parte de los demás, no seríamos ni siquiera capaces de tener fe en la forma en que aparecemos ante nosotros mismos"22.

El texto anterior pone de manifiesto que no solo el mundo común, sino nuestro conocimiento de quienes somos depende de que aparecemos ante los demás. Para Arendt el sensus communis es como un sexto sentido que coordina a los otros cinco y garantiza que estos responden a la percepción del mismo objeto, que los sentidos privados perciben lo que está en un mundo común que otros comparten. Como gozamos de cinco sentidos radicalmente distintos que tienen el mismo objeto común y todos los seres humanos estamos de acuerdo respecto a la identidad del mismo, la subjetividad se salva en cierto modo por esta comunidad en la percepción. De esta afinidad surge, señala Arendt, la "sensación de realidad" 23 .

La digitalización ataca directamente la raíz de esta sensación al buscar sustituir los sentidos. Los cambios digitales en la percepción no solo sustituyen cómo percibimos el mundo, sino cómo somos percibidos: ofrecen un mundo nuevo y un nuevo yo. Pasquale ha subrayado esta doble transformación epistemológica, esta doble deformación. La primera está caracterizada por las tecnologías de búsqueda (que median cómo percibimos), la segunda por las tecnologías de la reputación (que median cómo somos percibidos $)^{24}$.

Las tecnologías de la reputación determinan cómo somos percibidos en esta realidad deformada. Se enmarcan dentro de la sociedad de la puntuación en la que los algoritmos analizan la información y evalúan al individuo en cualquier ámbito y con cualquier fin, ya sea en relación al crédito, la salud, el trabajo o la inclusión en un fichero policial. Estas tecnologías sustituyen la historia personal por

\footnotetext{
${ }^{22}$ ARENDT, H.; La vida del espíritu (trad. R. Montoro Romero y F. Vallespín Oña), Madrid, CEC, 1984, p. 62.

${ }^{23}$ Ibid., p. 67.

${ }^{24}$ PASQUALE, F.; The Black Box Society, cit.
} 
información interpretada algorítmicamente. Sin embargo, aunque el individuo es desnudado por el algoritmo, las reglas que lo puntúan son opacas, no están reguladas y no reflejan un resultado carente de prejuicios sino que los multiplican ${ }^{25}$. Dan lugar, por ello, a diversas formas de discriminación racial, hacia el pobre, el enfermo o el disidente. Esta es la sociedad de clases digital basada en la puntuación, como ha visto Han, esta es la nueva forma de discriminación ${ }^{26}$. Cuando el gobierno entra en este ámbito surge la nación de la vigilancia que se caracteriza por la alianza público-privada en la que los datos se intercambian logrando una vigilancia del individuo cada día más completa y sin ángulos muertos $^{27}$.

Por su parte, las tecnologías de búsqueda van mucho más allá de los meros buscadores, producen el mundo que quieren enseñarnos, son Google, Amazon, Apple, Twitter, Facebook y todos los demás que median algorítimicamente cómo percibimos ${ }^{28}$. La deformación de las tecnologías de búsqueda no sustituye la realidad por otra, sino por múltiples realidades personalizadas ${ }^{29}$. El número de realidades que se presentan al individuo es virtualmente indefinido y se diluye el principio de nocontradicción. No se trata ya solo de personalizar por usuario, sino de personalizar según el instante mismo en que la tecnología presenta la realidad deformada. Una realidad deformada ya es negativa, mil realidades deformadas suponen una deformación monstruosa.

A su vez, la extracción de datos ayuda a mejorar esta doble deformación al mejorar la materia prima de los algoritmos que es la información. A mayor volumen, más precisión estadística. La vigilancia mejora a los vigilantes de forma exponencial y optimiza la deformación y el alejamiento de la realidad.

\subsection{Información formal frente a conocimiento y sabiduría}

Lo anterior pone de manifiesto que la segunda ilustración no mejora nuestro conocimiento, sino

que lo dificulta enormemente. Es verdad que supone el paso de la escasez a la abundancia de

\footnotetext{
${ }^{25}$ Ibid., pp. 19-58.

${ }^{26}$ HAN, B.-C.; Psicopolítica, cit., p. 99.

${ }^{27}$ PASQUALE, F.; The Black Box Society, cit., pp. 43-49.

${ }^{28}$ Ibid., p. 70.

${ }^{29}$ Ibid., pp. 60ss.
} 
información ${ }^{30}$. Sin embargo, paradójicamente, hace que el conocimiento sea más difícil que nunca por la personalización de la realidad, pero además porque la información se presenta de forma tan rápida que el cerebro humano no puede hacerse cargo de ella, tiene una temporalidad que dificulta enormemente la racionalidad y, por ello, el conocimiento. Además, la emocionalización conduce a que se promueva estructuralmente la información falsa como se comenta más adelante. A su vez, los datos recogidos gracias a esta realidad personalizada se ponen al servicio de los brókers de la información ${ }^{31}$.

La tecnología puede presentar información, pero el conocimiento requiere de un sujeto que seleccione y juzgue. Es importante la distinción de T.S. Eliot entre información, conocimiento y sabiduría, por niveles del más bajo al más alto de saber. Ya Postman, luego Han y, en España, Oliver, insisten en esta distinción ${ }^{32}$. Frente a esta distinción, el dominio de la ideología dataísta veda temas como la muerte o el sufrimiento por la profundidad que exigen ${ }^{33}$, a no ser que se pretenda ilusoriamente superarlos como hace la ideología transhumanista.

Tal es la sustitución del individuo por el algoritmo en el acceso a la realidad, que se pretende prescindir del teórico en todos los ámbitos del saber: el big data hace superfluo al teórico ${ }^{34}$. Ciertamente, la digitalización permite una predicción sin precedentes, mayor cuanto más gregario sea el hombre, pero es incapaz de aportar un sentido y una finalidad a la acción humana. Ya advirtió Arendt, con la que se comenzaba esta reflexión, que el científico se deshumaniza al mirar el mundo desde un lugar exterior, desde allí fácilmente confunde sus inventos tecnológicos con los seres humanos. Todo se hace homogéneo: lo dado y lo construido se confunden. Además, Arendt señala el peligro de la formalización del lenguaje, su matematización y la pérdida de sentido como característicos del cientificismo de entonces, rasgos aplicables al dataísmo actual:

"Todo nuestro orgullo por lo que podemos hacer desaparecerá en una especie de mutación de la raza humana; el conjunto de la tecnología, observado desde ese lugar, en realidad ya no se ve "como el

\footnotetext{
${ }^{30}$ FLORIDI, L. et al.; The Onlife Manifesto, cit., pp. $45 \mathrm{ss}$.

${ }^{31}$ CENTER FOR HUMANE TECNOLOGY; "Rock the Voter", Your Undivided Attention, Entrevista a Britanny Kaiser, 2019, p. 17.

${ }^{32}$ De los no citados anteriormente, véase, OLIVER, N.; "Inteligencia artificial: ficción, realidad... y sueños". Discurso de ingreso en la Real Academia de Ingeniería (11 de diciembre de 2018), p. 40. Sobre esto véase también BALLESTEROS, J.; "El futuro del derecho como lucha contra la idolatría tecnológica", Persona y derecho, núm. 79, 2018, pp. 37-50.

${ }^{33}$ HAN, B.-C.; La salvación de lo bello (trad. A. Ciria), Barcelona, Herder, 2015, p. 43.

${ }^{34}$ STEADMAN, I.; "Big Data and the Death of the Theorist", Wired, 25 enero de 2013.
} 
resultado de un esfuerzo humano consciente para extender los poderes materiales del hombre, sino más bien como un proceso biológico a gran escala". En tales circunstancias, el lenguaje y el habla cotidiana ya no serían una manifestación significativa que trasciende la conducta aunque sólo la exprese, y se podrían reemplazar con ventaja por el extremo, y en sí mismo no significativo, de los signos matemáticos" ${ }^{35}$.

\section{Deformación antropológica y política}

\subsection{Economía de la atención: expropiación, adicción y transparencia}

La tecnología digital tiene un enorme carácter parasitario, su lucro procede de las actividades de otros, es el registro de la vida del usuario lo que es objeto de lucro. El usuario de la tecnología digital es, al mismo tiempo, el "producto" con el que se comercializa. El expropiado es el usuario-producto. Arendt detecta precisamente como característico del capitalismo su carácter expropiador. Su pensamiento es, en este punto, del todo actual. Hoy se une el deseo crematístico del expropiador con el deseo de exposición y de reconocimiento del expropiado. El primero actúa de forma análoga a aquel que especula sobre el suelo y gana dinero a partir de la riqueza que rodea la propiedad (como denunciaba Henry George) $)^{36}$, los gigantes tecnológicos se lucran a partir de los millones de usuarios que pasan por sus redes ${ }^{37}$. De la expropiación de la esfera privada de la casa, característica del capitalismo original $^{38}$, hemos llegado a la pérdida de lo más íntimo que aquella esfera contenía, el pensamiento.

Lo inteligente de esta expropiación es que se logra, en apariencia, voluntariamente, aunque ciertamente la persuasión de la tecnología juega un papel esencial pues es adictiva por diseño ${ }^{39}$. Patrick Pharo considera que esta adicción es propia de la forma actual del capitalismo como un "proceso

\footnotetext{
${ }^{35}$ ARENDT, H.; Entre el pasado y el futuro. Ocho ejercicios sobre la reflexión politica (trad. A. Poljak), Barcelona, Península, 1996, p. 293.

${ }^{36}$ Citado por RAWORTH, K.; Doughnut Economics. Seven Ways to Think Like a $21^{\text {st }}$-Century Economist, Londres, Penguin Random House, 2017, p. 179.

${ }^{37}$ PASQUALE, F.; The Black Box Society, cit., p. 85.

${ }^{38}$ Remito a mi trabajo sobre la expropiación moderna de la esfera privada: "Hannah Arendt: from Property to Capital... and Back?", Archiv für Rechts- und Sozialphilosophie, $\mathrm{n}^{\circ}$ 2, 2018, pp. 184-201.

${ }^{39}$ CENTER FOR HUMANE TECNOLOGY; "Should've Stayed in Vegas", Your Undivided Attention, Entrevista a Natasha Schüll (2), 2019, pp. 1-17.
} 
adictivo" que funciona en los circuitos neuronales del placer y la recompensa para incrementar el deseo y reproducir indefinidamente los deseos ${ }^{40}$.

Si atendemos al carácter adictivo específico de la tecnología digital, este ecosistema tecnológico está producido a imagen de las máquinas tragaperras y el entorno de los casinos de Las Vegas. Se busca que el usuario olvide su propio cuerpo, anestesiar sus sentidos. Es la lógica del "círculo lúdico". Este requiere la soledad del individuo, el estímulo inmediato, la recompensa aleatoria y, por último, la carencia de límites ${ }^{41}$. Esta lógica la aplican las grandes tecnológicas que tienen como objetivo lograr la atención absoluta del usuario conforme al centro de su actividad económica que es la extracción de $\operatorname{datos}^{42}$.

Esta expropiación reduce al individuo a información que hay desvelar y explotar. Por ello, Han habla de la "sociedad pornográfica", considera que el mundo digital supone la total exposición de las personas hasta el inconsciente ${ }^{43}$. Se logra una adicción a la exposición, a que en la vida no haya ángulos muertos ni ante el otro-digital, ni ante el algoritmo que, de forma creciente, tiene información que el individuo desconoce. Se logra una "economía de la vida integral"44.

Además, como ha visto Gloria Mark, las consecuencias de la adicción para un bien humano fundamental, la concentración y el tiempo de atención continuada para realizar una tarea, son enormes $^{45}$. Según Pasquale, lo que Wall Street hace con el dinero, Sillicon Valley lo hace con nuestra atención.

Esta adicción y la pérdida de la concentración necesitan de la soledad, aunque sea la soledad de estar "solos juntos", de ignorar al que está físicamente al lado. La soledad como falta de relaciones significativas es, en este punto, enormemente lucrativa. Pero, ciertamente, la soledad es la semilla de

\footnotetext{
${ }^{40}$ Un proceso que afecta al consumo, producción, vigilancia o especulación financiera. Véase su obra PHARO, P.; Le capitalisme addicctif, Editions PUF, 2018.

${ }^{41}$ CENTER FOR HUMANE TECNOLOGY; "Should've Stayed in Vegas”, cit., pp. 9ss.

${ }^{42}$ La economía de la extracción de la atención no es igual en todas las tecnológicas. Apple, por ejemplo, no tiene como centro de su actividad la recogida de datos. Tristan Harris en CENTER FOR HUMANE TECNOLOGY; "Should've Stayed in Vegas", cit., p 13.

${ }^{43}$ HAN, B.-Y.; Psicopolítica, cit., p. 96. Hemos desarrollado este tema en "Título" (2018).

${ }^{44}$ SADIN, E.; La silicolonizacion del mundo, cit., p. 28.

${ }^{45}$ CENTER FOR HUMANE TECNOLOGY; "Pardon for the Interruptions”, Your Undivided Attention, Entrevista a Gloria Mark, 2019.
} 
muchos males, como ya señaló Arendt, al considerar el totalitarismo como "la soledad organizada"46. La tecnología digital no puede sustituir las relaciones significativas ni construir un orden común, sino “organizar la soledad”.

Cuanto más se vuelve uno a su mundo particular digital más y mejor se explota toda su vida humana. Despertar es la pérdida que la "silicolonización"47 no puede admitir. La pérdida de la atención y de la voluntad son algunas de las consecuencias de la tecnología digital, pero esta realidad deformada también imposibilita distinguir la verdad de la mentira y deteriora lo político al promover la polarización, el voto dirigido y la eliminación de la disidencia, entre otras cosas.

\section{2. ¿Libertad de expresión o multiplicación gratuita?}

La segunda ilustración no solo ha defraudado las expectativas para nuestro conocimiento y para nuestras relaciones humanas, también ha defraudado las expectativas políticas. Las esferas informales de la política no han resultado ser ni informales (por estar dirigidas), ni políticas (por deteriorar lo común y político). La tecnología digital deforma la política al dañar la integridad de las elecciones, difundir la mentira, fomentar el odio, polarizar el discurso, permitir la manipulación extranjera, permitir eliminar al disidente mediante mentiras $y$, en general, rebajar la racionalidad política a emocionalidad política ${ }^{48}$.

En las redes sociales domina una forma antipolítica de expresión, la emoción, que va unida a la aceleración. A mayor aceleración más dominio de la emoción. La emoción es dinámica y situacional, frente a la racionalidad que es estable y lenta ${ }^{49}$. Dentro de esta emocionalización a nivel pre-reflexivo los algoritmos dan primacía a aquello que estadísticamente logra una mayor adicción. La tecnología digital es antipolítica porque multiplica todo lo irracional y devalúa la libertad de expresión convertida en multiplicación gratuita para algunos o en simple libertad de la emoción para otros.

\footnotetext{
${ }^{46}$ ARENDT, H.; Los orígenes del totalitarismo (trad. G. Solana), Madrid, Alianza, 2011, p. 640.

${ }^{47}$ SADIN, E.; La silicolonizacion del mundo, cit.

${ }^{48}$ CENTER FOR HUMANE TECNOLOGY; "Rock the Voter”, cit., p. 11. Véase también MARTÍNEZ OTERO, J.; "Gatitos contra la democracia... (o de cómo las redes sociales pueden minar nuestro sistema de convivencia)" en Para una nueva cultura política (A. Masferrer, ed.), Madrid, Catarata, 2019, pp. 174-177.

${ }^{49}$ HAN, B.C.; Psicopolítica, cit., pp. 65ss.
} 
Como la emoción domina y no atiende a razones, las informaciones no están en pie de igualdad, la libertad de expresión no es, en absoluto, igual para todos. En las redes sociales la mentira, el insulto o la negación de una realidad evidente son potenciados por los algoritmos porque ese contenido permite acrecentar la atención de los usuarios. Dado lo anterior, los usuarios que ofrecen esta información deformada no solo disfrutan de la libertad de expresión sino de una ventaja adicional: la de la multiplicación. Renée DiResta ha establecido esta distinción entre la libertad de expresión que es igual para todos y la libertad de multiplicación, o multiplicación gratuita, que es la que otorgan los algoritmos a aquellos que comparten contenidos que técnicamente funcionan ${ }^{50}$. Así ocurre, por ejemplo, con la tendencia de las recomendaciones de Youtube que están diseñadas para llevarnos al huerto por diseño ${ }^{51}$.

Esta multiplicación permite nuevas formas de propaganda y manipulación política por muy poco dinero y con un alcance amplísimo con solo dominar unas pocas plataformas ${ }^{52}$. Es posible una “micro-focalización” muy personalizada y extraordinariamente difícil de detectar. Se puede "manufactuar el consenso" en distintas plataformas. Por ejemplo, mediante los trending topics en Twitter que se basan en la velocidad de la expansión de los mensajes, no en su popularidad. El algoritmo no puede tener una estructura menos política ni menos humana, da total primacía a la rapidez. No es raro, entonces, que se empleen bots automatizados para "manufacturar una verdad" haciéndola tendencia. Los bots son mucho más rápidos que los seres humanos para lograr un trending topic $^{53}$. En cambio, posiciones políticas como Ocuppy Wall Street, a pesar de su enorme seguimiento y popularidad, no son tendencia para sorpresa de muchos ${ }^{54}$. ¿El problema? Su lentitud en ser populares o, en otros términos, su mayor racionalidad y temporalidad humanas.

Con lo anterior, el lema que afirma "si lo puedes hacer tendencia, lo puedes hacer verdad" se cumple. En todas las plataformas la extraordinaria simplicidad de la comunicación permite una extensión rápida y un aparente consenso muy homogéneo que sería imposible alrededor de ideas

\footnotetext{
50 Para mantener la forma rimada, se usa "multiplicación" (reach) con "expresión" (speech). Véase CENTER FOR HUMANE TECNOLOGY; "From Russia with Likes", Your Undivided Attention, Entrevista a Renée DiResta (1), pp. 7ss.

${ }^{51}$ En inglés: "Down the Rabbit Hole by Dessign". Véase también PASQUALE, F.; The Black Box Society, cit., p. 73. Por ejemplo, los videos sobre la reserva federal conducen a recomendaciones de vídeos antisemitas.

52 CENTER FOR HUMANE TECNOLOGY; "From Russia with Likes", cit., p. 10.

${ }^{53}$ Ibid., pp. 2-5.

${ }^{54}$ PASQUALE, F.; The Black Box Society, cit., p. 76.
} 
complejas. Dada su superficialidad y rápida extensión la herramienta preferida es el meme ${ }^{55}$, pero puede ser cualquier otra, como un pequeño texto o un vídeo. Frente a esta herramienta es lógico que la corrección a la información, en el caso de informaciones falsas, nunca se extienda como la información original $^{56}$. Si la tecnología digital es un medio rápido y emocional estas correcciones apenas sirven por oponerse a la propia estructura de la tecnología.

Un caso singularmente inteligente de manipulación electoral es el que instrumentaliza la identidad del individuo, método empleado por la inteligencia rusa para favorecer a Trump. La manipulación electoral aquí es indistinguible de los contenidos realmente creados por los grupos, lo que la hace virtualmente indetectable para el usuario ${ }^{57}$. La manipulación política consiste en detectar la identidad de los grupos sociales, crear cuentas de Twitter falsas que promueven esa identidad, conseguir seguidores, alimentarla mediante una información que refuerza dicha identidad y hacer palanca en el momento adecuado cerca de las elecciones en una dirección u otra. Esto supone una propaganda electoral que rechaza el principio de no-contradicción pues se pueden estar ofreciendo mensajes radicalmente opuestos a grupos distintos con publicidad contradictoria según el usuario al que se destina. Así ocurrió en la campaña Trump cuya innovación fue su carácter completamente peronalizado y negativo ${ }^{58}$. Entre los votantes o posibles votantes de Trump se promueve el odio y el enfado. Por otro lado, entre grupos que podrían votar frente a Trump se procura hacerlos apáticos para que no voten como, por ejemplo, a los negros a los que se persuade de que no son ciudadanos del país ${ }^{59}$.

Pero el caso Trump o el Brexit solo son la punta del iceberg, Cambridge Analytica llevó la campaña electoral mediante la manipulación personalizada o "micro-focalización" en cincuenta países $^{60}$. Los países menos poderosos se encuentran mucho más indefensos ante esto. En Filipinas,

\footnotetext{
${ }^{55}$ CENTER FOR HUMANE TECNOLOGY; "From Russia with Likes", cit., pp. 4-9.

${ }^{56}$ Ibid., p. 6.

${ }^{57}$ Ibid., pp. 14-18.

${ }^{58}$ CENTER FOR HUMANE TECNOLOGY; "Rock the Voter", cit., p. 6.

${ }^{59}$ CENTER FOR HUMANE TECNOLOGY; "From Russia with Likes", cit., p. 17.

${ }^{60}$ CENTER FOR HUMANE TECNOLOGY; "Rock the Voter", cit., p. 14
} 
donde la práctica totalidad de la población está en Facebook, se elimina el periodismo objetivo mediante mentiras que el propio presidente, Rodrigo Duterte, manufactura en las redes sociales ${ }^{61}$.

Buena parte de estos problemas se vuelve mayor en la medida en que las plataformas tienen carácter global. Es necesario cuestionar la pretensión de las plataformas de constituir una "esfera pública global"62. Frente a ella se puede recordar a Arendt que considera que la libertad solo surge en pequeñas esferas de poder $^{63}$. Si admitimos que cabe algún tipo de política en internet serán necesarios pequeños espacios de diferente tamaño ${ }^{64}$.

\section{3. ¿Inteligibilidad o cajas negras?}

Ciertamente, el poder no está en manos del usuario sino de los diseñadores de Sillicon Valley. Byung-Chul Han considera "transparencia" la palabra clave en la segunda ilustración, pero esta afecta sobre todo al individuo, no al poder. Para el poder la palabra es "caja negra" como ha visto Pasquale ${ }^{65}$. El poder algorítmico es opaco como ya se afirma comúnmente, bancos de inversión y tecnológicas dominan el mundo sin que podamos comprenderlo. Si la política requiere publicidad, el dominio, en cambio, requiere secreto. El engaño, la duplicación y el dominio de las apariencias son característicos de los regímenes totalitarios ${ }^{66}$.

Según Pasquale, en buena medida, la opacidad procede de una mala regulación de la propiedad intelectual, de la excesiva protección de determinadas patentes ${ }^{67}$. Otros señalan que la opacidad algorítmica puede ser opacidad intencional, por ignorancia o intrínseca ${ }^{68}$.

El secretismo y la incomprensión no permiten solo crear relaciones asimétricas, sino una auténtica veneración seudoreligiosa. La magia, dice Postman, dirige nuestra atención al lugar

\footnotetext{
${ }^{61}$ Véase CENTER FOR HUMANE TECNOLOGY; “The Dictator's Playbook”, Your Undivided Attention, Entrevista a Maria Ressa, 2019, p. 7. Los silliconianos también gozan de una posición política propia, pero es muy difícil de conocer. PASQUALE, F.; The Black Box Society, cit., pp. 73-76.

${ }^{62}$ CENTER FOR HUMANE TECNOLOGY; “From Russia with Likes”, p. 19; “To Russia with Likes (Part 2)”, 2019 , p. 31.

63 ARENDT, H.; Sobre la revolución, (trad. P. Bravo), Madrid, Alianza, 1998, 2a reempr. 2009, p. 203.

${ }^{64}$ CENTER FOR HUMANE TECNOLOGY; "From Russia with Likes", cit., p. 19.

${ }^{65}$ PASQUALE, F.; The Black Box Society, cit., pp. 6-7.

${ }^{66}$ ARENDT, H.; Los orígenes del totalitarismo, cit., pp. 324 ss., y p. 510.

${ }^{67}$ Ibidem.

${ }^{68}$ BURREL, J.; "How the machine 'thinks': Understanding opacity in machine learning algorithms", Big Data and Society, 2016, pp. 112.
} 
equivocado, por eso produce asombro y no comprensión ${ }^{69}$. La tecnología digital no muestra sus miserias al lego, sino sus logros y sus deseos de obrar por el bien de la humanidad ${ }^{70}$. La opacidad que oculta los mecanismos facilita la idolatría totémica hacia la tecnología digital ${ }^{71}$.

\section{A modo de conclusiones}

La tecnología digital actual rebaja la estatura del hombre. Se presentan aquí tres deformaciones operadas por la tecnología digital: epistemológica, antropológica y socio-política.

La deformación epistemológica dificulta el acceso a la realidad al sustituir los sentidos por información tratada algorítmicamente. El sentido común, como aquel sentido que engloba la información de los cinco sentidos en una comunidad de seres sensibles, queda seriamente perjudicado. En particular, las tecnologías de búsqueda permiten una personalización completa de los contenidos digitales que sustituyen la realidad sensible común. La emoción, que es performativa y lleva a estar digitalmente activo, permite que la comunicación y la información se multipliquen exponencialmente.

La segunda deformación es la antropológica. La emocionalización del medio digital permite la adicción del usuario. Las tecnologías de búsqueda logran captar la atención cada vez con más éxito y multiplicar cada vez más el tiempo en el dispositivo de los usuarios. Las grandes empresas tecnológicas se lucran de la copia digital que obtienen de la vida del usuario, una vida registrada en un número cada vez más creciente de actividades.

La tercera deformación es la socio-política. Por un lado, la tecnología digital refuerza el fenómeno de la soledad. El apartamiento de la realidad y la comunicación emocional dificultan el nacimiento de relaciones significativas. Por otro lado, el imperativo económico de optimizar el tiempo en el dispositivo de los usuarios provoca que lo falso, lo pornográfico o lo que polariza a la sociedad goce del privilegio de la llamada libertad de multiplicación. Los contenidos de esta índole son multiplicados por los algoritmos porque son el contenido que funciona más adecuadamente desde el punto de vista económico. Cuando este problema estructural se aprovecha políticamente se puede

${ }^{69}$ POSTMAN, N.; Tecnópolis, cit., p. 131.

${ }^{70}$ SADIN, E.; La silicolonización del mundo, cit., pp. 97ss.

${ }^{71}$ SADIN, E.; La humanidad aumentada, cit., p. 90. 
manipular a un número extraordinario de electores sin que sean conscientes en absoluto de dicha manipulación.

Esta triple deformación de la realidad requiere una transformación de la tecnología digital y de nuestra relación con ella. Es posible una tecnología digital distinta, puesta al servicio del ser humano y no una herramienta de dominio.

El camino a seguir pasa por recuperar el gusto por los sentidos tal y como nos han sido dados, así como la capacidad de admiración y de reflexión que exigen lentitud. La aceptación de la realidad se presenta adecuadamente con el modelo del jardinero que puede abrir una brecha en la deformación tecnológica. El jardinero se distingue de sus instrumentos y del jardín al gozar de conocimiento sensible. Acepta que el jardín solo se puede conocer y cuidar respetando su otredad, que no se deja someter ni al cálculo algorítmico ni a la emoción pasajera. 


\section{Bibliografía}

ARENDT, H.; La vida del espíritu (trad. R. Montoro Romero y F. Vallespín Oña), Madrid, CEC, 1984.

ARENDT, H.; Entre el pasado y el futuro. Ocho ejercicios sobre la reflexión política (trad. A. Poljak), Barcelona, Península, 1996.

ARENDT, H.; Sobre la revolución, (trad. P. Bravo), Madrid, Alianza, 1998, 2ª reempr. 2009.

ARENDT, H.; Los orígenes del totalitarismo (trad. G. Solana), Madrid, Alianza, 2011.

BALLESTEROS, A.; "Hannah Arendt: from Property to Capital... and Back?", Archiv für Rechts- und Sozialphilosophie, $\mathrm{n}^{\mathrm{o}}$ 2, 2018, pp. 184-201.

BALLESTEROS, J.; "El futuro del derecho como lucha contra la idolatría tecnológica”, Persona y derecho, núm. 79, 2018, pp. 37-50.

BURREL, J.; "How the machine 'thinks': Understanding opacity in machine learning algorithms", Big Data and Society, 2016.

CENTER FOR HUMANE TECNOLOGY; "Should've Stayed in Vegas", Your Undivided Attention, Entrevista a Natasha Schüll (2), 2019.

CENTER FOR HUMANE TECNOLOGY; "Down the Rabbit Hole by Dessign", Your Undivided Attention, Entrevista a Guillaume Chaslot, 2019.

CENTER FOR HUMANE TECNOLOGY; "From Russia with Likes", Your Undivided Attention, Entrevista a Renée DiResta (1), 2019.

CENTER FOR HUMANE TECNOLOGY; "To Russia with Likes" (Part 2), Your Undivided Attention, Entrevista a Renée DiResta (2), 2019.

CENTER FOR HUMANE TECNOLOGY; "Pardon for the Interruptions", Your Undivided Attention, Entrevista a Gloria Mark, 2019.

CENTER FOR HUMANE TECNOLOGY; “The Dictator's Playbook”, Your Undivided Attention, Entrevista a Maria Ressa, 2019.

CENTER FOR HUMANE TECNOLOGY; "Rock the Voter", Your Undivided Attention, Entrevista a Britanny Kaiser, 2019.

DEWANDRE, N.; "The Human Condition and The Black Box Society", https://www.boundary2.org/2015/12/dewandre-on-pascal/ [consultado:18/12/2019].

FLORIDI, L. (ed.) et al.; The Onlife Manifesto. Being Human in a Hyperconnected Era, Springer Open, 2015.

HAN, B.-C.; En el enjambre (trad. R. Gabás), Barcelona, Herder, 2014.

HAN, B.-C.; Psicopolítica. Neoliberalismo y nuevas técnicas de poder (trad. A. Bergés), Barcelona, Herder, 2014.

HAN, B.-C.; La salvación de lo bello (trad. A. Ciria), Barcelona, Herder, 2015.

LASALLE, J. M.; Ciberleviatán, Barcelona, Arpa, 2019.

MASFERRER, A. (ed.) et al.; Para una nueva cultura política, Madrid, Catarata, 2019.

McLUHAN, M.; Comprender los medios de comunicación. Las extensiones del ser humano (trad. P. Ducher), Barcelona, Paidós, 1996. 
MORATALLA, N.; Inteligencia artificial. ¿Conciencia artificial? Madrid, Digital Reasons, 2017.

OLIVER, N.; “Inteligencia artificial: ficción, realidad... y sueños”. Discurso de ingreso en la Real Academia de Ingeniería (11 de diciembre de 2018).

PARISER, E.; El filtro burbuja. Cómo la red decide lo que leemos y lo que pensamos (trad. M. Vaquero), Taurus, 2017.

PASQUALE, F.; "Platform Neutrality: Enhancing Freedom of Expression in Spheres of Private Power", Theoretical Inquiries in Law, vol. 17, 2016

PHARO, P.; Le capitalisme addicctif, Editions PUF, 2018.

POSTMAN, N.; Tecnópolis. La rendición de la cultura a la tecnología (trad. A. Almazán y S. Miras), Ediciones El salmón, 2018.

RAWORTH, K.; Doughnut Economics. Seven Ways to Think Like a $21^{\text {st }}$-Century Economist, Londres, Penguin Random House, 2017.

SADIN, E.; La humanidad aumentada (trad. J. Blanco y C. Paccazochi), Buenos Aires, Caja negra, 2017.

SADIN, E.; La silicolonización del mundo. La irresistible expansión del liberalismo digital (trad. M. Martínez), Buenos Aires, Caja negra, 2018.

STEADMAN, I.; "Big Data and the Death of the Theorist", Wired, 25 enero de 2013.

TURKLE, S.; Alone Together. Why We Expect More from Technology and Less from Each Other, New York, Basic Books, 2011.

VERBEEK, P.-P.; Understanding and Designing the Morality of Things, Chicago, University of Chicago Press, 2011.

WU, T.; The Attention Merchants' Dissects the Battle for Clicks and Eyeballs, Random House, 2016. 